\title{
Bacterial nanocellulose applications for tissue engineering
}

\begin{abstract}
Nanocellulose is one of the most promising natural polymers to substitute conventional polymers currently employed for tissue engineering applications. The three different types of nanocellulose (cellulose nanocrystals, cellulose nanofibrils, and bacterial cellulose) are presented in this chapter. However, the main focus of discussion is bacterial cellulose (BC) for tissue engineering applications, owing to its meritorious properties such as physical (high purity, permeability, water absorption capacity, and porosity), mechanical (high tensile strength), and biological properties (good biocompatibility and biodegradability). These physical, biological, and mechanical properties of $\mathrm{BC}$ are features that enable $\mathrm{BC}$ membranes to function as effective temporary wound dressing biomaterial compared with conventional wound dressing materials. BC membranes can easily absorb exudate during wound dressing process and are smoothly removed from a wound surface after recovery. BC used for permanent implantation can remain in the body without causing any toxic or inflammatory reactions due to its good biocompatibility properties. In addition, BC membranes are developed in any shape and size, which enhance their suitability to cover large and difficult areas of the body. Hence, interest in BC biofabricated materials has accelerated steadily as a result of their remarkable potential usage in tissue engineering applications.
\end{abstract}

Keyword: Bacterial cellulose; Nanocrystals; Nanofibrils; Nanocellulose; Tissue engineering 